\title{
Bayesian Position Estimation of an Industrial Robot using Multiple Sensors
}

\author{
Rickard Karlsson, Mikael Norrlöf \\ Control \& Communication \\ Department of Electrical Engineering \\ Linköpings universitet, SE-581 83 Linköping, Sweden \\ WWW: http://Www. control.isy.liu.se \\ E-mail: rickard@isy.liu.se, mino@isy.liu.se
}

8th June 2004

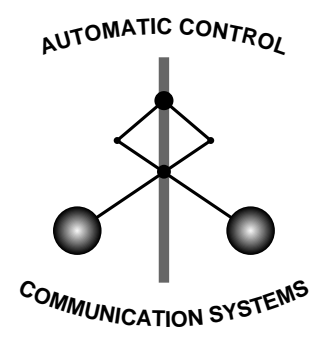

LINKÖPING

Report no.: LiTH-ISY-R-2613

Submitted to IEEE Conference on Control Applications 2004, Taipei, Taiwan

Technical reports from the Control \& Communication group in Linköping are available at http://www. control.isy.liu.se/publications. 


\begin{abstract}
A modern industrial robot control system is often only based upon measurements from the motors of the manipulator. To perform good trajectory tracking on the arm side of the robot a very accurate description of the system must therefore be used. In the paper a sensor fusion technique is presented to achieve good estimates of the position of the robot using a very simple model. By using information from an accelerometer at the tool of the robot the effect of unmodelled dynamics can be measured. The estimate of the tool position can be improved to enhance accuracy. We formulate the computation of the position as a Bayesian estimation problem and propose two solutions. The first solution uses the extended Kalman filter (EKF) as a fast but linearized estimator. The second uses the particle filter which can solve the Bayesian estimation problem without linearizations or any Gaussian noise assumptions. Since the aim is to use the positions estimates to improve position with an iterative learning control method, no computational constraints arise. The methods are applied to experimental data from an ABB IRB1400 commercial industrial robot and to data from a simulation of a realistic flexible robot model, showing a significant improvement in position accuracy.
\end{abstract}

Keywords: extended kalman filter, particle filter, robotics, accelerometer 


\title{
Bayesian Position Estimation of an Industrial Robot using Multiple Sensors
}

\author{
Rickard Karlsson and Mikael Norrlöf
}

\begin{abstract}
A modern industrial robot control system is often only based upon measurements from the motors of the manipulator. To perform good trajectory tracking on the arm side of the robot a very accurate description of the system must therefore be used. In the paper a sensor fusion technique is presented to achieve good estimates of the position of the robot using a very simple model. By using information from an accelerometer at the tool of the robot the effect of unmodelled dynamics can be measured. The estimate of the tool position can be improved to enhance accuracy. We formulate the computation of the position as a Bayesian estimation problem and propose two solutions. The first solution uses the extended Kalman filter (EKF) as a fast but linearized estimator. The second uses the particle filter which can solve the Bayesian estimation problem without linearizations or any Gaussian noise assumptions. Since the aim is to use the positions estimates to improve position with an iterative learning control method, no computational constraints arise. The methods are applied to experimental data from an ABB IRB1400 commercial industrial robot and to data from a simulation of a realistic flexible robot model, showing a significant improvement in position accuracy.
\end{abstract}

\section{INTRODUCTION}

Modern industrial robot control is usually based upon measurements from only the motor angles of the manipulator. The ultimate goal however is to make the tool move according to some predefined path. In [10] a method for improving the absolute accuracy of a standard industrial manipulator is described. The improved accuracy is achieved through

- Identification of unknown or uncertain parameters in the robot system.

- Using additional sensors.

- Applying the iterative learning control (ILC) method, [2], [15].

An example of one possible external measurement system characterized by high accuracy but also high price is the laser tracker from Leica Geosystems, [7]. This system will later on be used to evaluate the technique proposed in this paper. The aim of this paper is to present a solution where the rather expensive laser tracker is not needed in the control loop. Instead the improved accuracy is achieved by using an inexpensive 3 degrees-of-freedom (DOF) accelerometer, [5], and Bayesian estimation techniques. We apply our

This work was supported by the VINNOVA competence center ISIS at Linköping University

R. Karlsson and M. Norrlöf are both with the Dept. of Electrical Engineering, Linköping University, SE-58183 Linköping, Sweden. The e-mail addresses to the authors are rickardeisy.liu.se and mino@isy.liu.se, respectively.

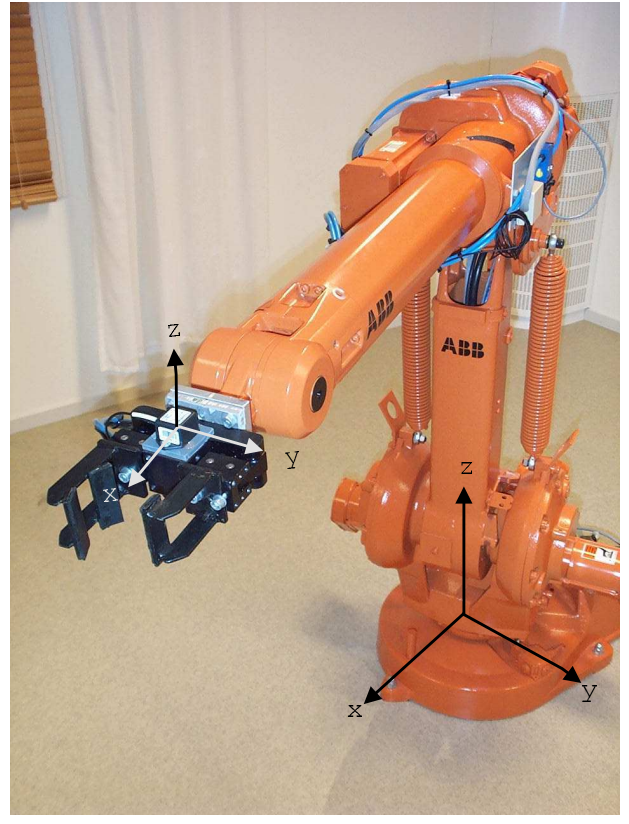

Fig. 1. The ABB IRB1400 robot with the Crossbow CXL02LF3 accelerometer. The base coordinate system and the coordinate system for the accelerometer are also shown.

method to experimental data from an ABB IRB1400 commercial industrial robot, shown in Fig 1, and to data from a realistic flexible robot model.

Traditionally, estimation problems are solved using linearized filters, mainly extended Kalman filters (EKF) [1]. The robot dynamics and measurements are highly nonlinear and the measurement noise is not always Gaussian. Hence, linearized models may not always be a good approach. The particle filter (PF),[6], provides a general solution to many problems where linearizations and Gaussian approximations are intractable or would yield too low performance.

\section{Motivations}

With a highly accurate tool position estimate, the control of the robot can be improved. However, to incorporate the estimates in a closed loop real-time system may not be possible due to the computational complexity in the estimation methods. This is not a problem in some practical applications. Consider for instance Iterative Learning Control (ILC), which is an off-line method. ILC has over the years become a standard method for achieving high 
accuracy in industrial robot control [2], [17], [18]. It utilizes a repetitive system dynamics to compensate for errors. Mathematically an ILC control law can be written as

$$
u_{t, k+1}=\mathcal{Q}\left(u_{t, k}+\mathcal{L} \epsilon_{t, k}\right),
$$

where $u_{t, k}$ is the ILC input in the $k$ th iteration and $\epsilon_{t, k}$ is the error. The error is defined as $\epsilon_{t, k}=r_{t}-y_{t, k}$ where $r$ is the reference and $y_{t, k}$ the measured output of the system. $\mathcal{Q}$ and $\mathcal{L}$ are design parameters for the control law. In industrial robot systems the measured output does not correspond to the actual controlled output. An ILC experiment on the ABB IRB1400 in [16, Chapter 9] using only motor angle measurements, i.e., no accelerometer, shows that although the error on the motor-side is reduced the path on the armside does not follow the programmed path. This is illustrated in Fig 2.
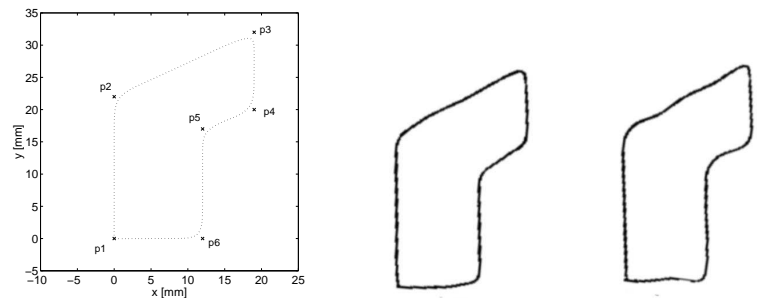

Fig. 2. Results from an ILC experiment on the ABB IRB1400 robot where ILC is applied using only motor angle measurements. Programmed path (left), iteration 0 (middle), and iteration 10 (right).

The idea in this paper is to use an accelerometer on the tool to get measurements that reflects the actual tool motion, see Fig 1. From these measurements and a model of the robot the position error, $\hat{\epsilon}_{t, k}$, is estimated and used in the ILC update equation according to

$$
u_{t, k+1}=\mathcal{Q}\left(u_{t, k}+\mathcal{L} \hat{\epsilon}_{t, k}\right) .
$$

In [11] a 1 DOF lab-process is controlled using this technique but the estimation is simplified compared to the approach suggested in this paper due to some assumptions on the system.

\section{BAYESIAN ESTIMATION}

Consider the discrete state-space model

$$
\begin{aligned}
x_{t+1} & =f\left(x_{t}, u_{t}, w_{t}\right), \\
y_{t} & =h\left(x_{t}, e_{t}\right),
\end{aligned}
$$

with state variables $x_{t} \in \mathbb{R}^{n}$, input signal $u_{t}$ and measurements $\mathbb{Y}_{t}=\left\{y_{i}\right\}_{i=1}^{t}$, with known probability density functions (pdfs) for the process noise, $p_{w}(w)$, and measurement noise $p_{e}(e)$. The non-linear prediction density $p\left(x_{t+1} \mid \mathbb{Y}_{t}\right)$ and filtering density $p\left(x_{t} \mid \mathbb{Y}_{t}\right)$ for the Bayesian inference, [12], is given by

$$
\begin{aligned}
p\left(x_{t+1} \mid \mathbb{Y}_{t}\right) & =\int_{\mathbb{R}^{n}} p\left(x_{t+1} \mid x_{t}\right) p\left(x_{t} \mid \mathbb{Y}_{t}\right) d x_{t} \\
p\left(x_{t} \mid \mathbb{Y}_{t}\right) & =\frac{p\left(y_{t} \mid x_{t}\right) p\left(x_{t} \mid \mathbb{Y}_{t-1}\right)}{p\left(y_{t} \mid \mathbb{Y}_{t-1}\right)}
\end{aligned}
$$

These equations are in general not analytically solvable. However, for the important special case of linear-Gaussian dynamics and linear-Gaussian observations the Kalman filter, [13], will give the solution. For a general non-linear or non-Gaussian system approximate methods must be applied. Here we will consider two different approaches of solving the Bayesian equations, extended Kalman filter (EKF), and particle filter (PF). The EKF will solve the problem using a linearization of the system and assuming Gaussian noise. The particle filter on the other hand will approximately solve the Bayesian equations by stochastic integration. Hence, no linearizations errors occur. The particle filter can also handle non-Gaussian noise models where the pdfs are known only up to a normalization constant. Also hard constraints on the state variables can be incorporated in the estimation without any problems.

\section{A. The Extended Kalman Filter (EKF)}

For the special case of linear dynamics, linear measurements and additive Gaussian noise the Bayesian recursions in Section III have an analytical solution, the Kalman filter. For many non-linear problems the noise assumptions are such that a linearized solution will be a good approximation. This is the idea behind the EKF, [1], where the model is linearized around the previous estimate. Here we only briefly present the time- and measurement update for the EKF,

$$
\begin{aligned}
& \left\{\begin{array}{l}
\hat{x}_{t+1 \mid t}=f\left(\hat{x}_{t \mid t}, u_{t}\right), \\
P_{t+1 \mid t}=F_{t} P_{t \mid t} F_{t}^{T}+Q_{t},
\end{array}\right. \\
& \left\{\begin{array}{l}
\hat{x}_{t \mid t}=\hat{x}_{t \mid t-1}+K_{t}\left(y_{t}-h\left(\hat{x}_{t \mid t-1}\right)\right), \\
P_{t \mid t}=P_{t \mid t-1}-K_{t} H_{t} P_{t \mid t-1}, \\
K_{t}=P_{t \mid t-1} H_{t}^{T}\left(H_{t} P_{t \mid t-1} H_{t}^{T}+R_{t}\right)^{-1},
\end{array}\right.
\end{aligned}
$$

where we use the linearized matrices

$$
F_{t}=\left.\nabla_{x} f\left(x_{t}\right)\right|_{x_{t}=\hat{x}_{t \mid t-1}}, H_{t}=\left.\nabla_{x} h\left(x_{t}\right)\right|_{x_{t}=\hat{x}_{t \mid t-1}} .
$$

The noise covariances are given as

$$
Q_{t}=\operatorname{Cov}\left(w_{t}\right), R_{t}=\operatorname{Cov}\left(e_{t}\right) .
$$

\section{B. The Particle filter $(P F)$}

In this section the presentation of the particle filter theory is according to [3], [6], [8], [14]. The particle filter provides an approximative solution to the discrete time Bayesian estimation problem formulated in (4) by updating an approximative description of the posterior filtering density. Let $x_{t}$ denote the state of the observed system and $\mathbb{Y}_{t}=$ $\left\{y^{(i)}\right\}_{i=1}^{t}$ be the set of observed measurements until present time. The particle filter approximates the density $p\left(x_{t} \mid \mathbb{Y}_{t}\right)$ by a large set of $N$ samples (particles), $\left\{x_{t}^{(i)}\right\}_{i=1}^{N}$, where each particle has an assigned relative weight, $\gamma_{t}^{(\bar{i})}$, chosen such that all weights sum to unity. The location and weight of each particle reflect the value of the density in the region of the state space, The particle filter updates the particle location and the corresponding weights recursively with 
each new observed measurement. For the common special case of additive measurement noise, i.e.,

$$
y_{t}=h\left(x_{t}\right)+e_{t}
$$

the unnormalized weights are given by

$$
\gamma_{t}^{(i)}=p_{e}\left(y_{t}-h\left(x_{t}^{(i)}\right)\right), \quad i=1, \ldots, N
$$

Using the samples (particles) and the corresponding weights the Bayesian equations can be approximately solved. To avoid divergence a resampling step is introduced. This is referred to as the Sampling Importance Resampling (SIR), [8], and is summarized in Algorithm 1.



As the estimate for each time we chose the minimum mean square estimate, i.e.,

$$
\hat{x}_{t}=\mathbb{E}\left\{x_{t}\right\}=\int_{\mathbb{R}^{n}} x_{t} p\left(x_{t} \mid \mathbb{Y}_{t}\right) d x_{t} \approx \sum_{i=1}^{N} w_{t}^{(i)} x_{t}^{(i)} .
$$

Methods to handle outliers in the measurement data also exists but will not be explained here.

\section{The Estimation Model}

A general estimation problem consists of a non-linear state equation and a non-linear measurement relation (3) where the process noise, $w_{t}$, and measurement noise, $e_{t}$, are non-Gaussian, describing the object and measurement. Often additive noises are assumed. A common assumption of the dynamics of the robot is that the transmission can be approximated with two or three masses connected by springs. The coefficients are then estimated from an identification experiment. See for instance [20]. Here it will be assumed that the transmission can be described by a two mass system and that the manipulator is rigid. The equation describing the torque balance for the motor becomes

$$
\begin{array}{r}
M_{m} \ddot{q}_{m}=-f_{m} \dot{q}_{m}-r_{g} k\left(r q_{m}-q_{a}\right) \\
-r_{g} d\left(r \dot{q}_{m}-\dot{q}_{a}\right)+u
\end{array}
$$

where $M_{m}$ is the motor inertia matrix, $q_{m}$ the motor angle, $q_{a}$ the arm angle, $r_{g}$ the gear ratio, $f_{m}, k, d$ the motor friction, spring constant and damping respectively. Input to the system is the motor torque $u$. The corresponding relation for the arm becomes a non-linear equation

$$
\begin{array}{r}
M_{a}\left(q_{a}\right) \ddot{q}_{a}+C\left(q_{a}, \dot{q}_{a}\right) \dot{q}_{a}+g\left(q_{a}\right)= \\
k\left(r_{g} q_{m}-q_{a}\right)+d\left(r_{g} \dot{q}_{m}-\dot{q}_{a}\right) .
\end{array}
$$

The goal here is to estimate the arm position, $q_{a}$, by measuring the motor angle, $q_{m}$, and the Cartesian tool acceleration. The approach is similar to the one suggested in [9], [11], which uses the relation given by (12) in a case when the system is scalar and linear. The results presented here generalizes [9], [11] since a multivariable non-linear system is considered. To simplify the estimation model, (12) is used under the assumption that the motor and arm velocities are zero, i.e.,

$$
q_{m}=\frac{1}{r_{g}}\left(q_{a}+\frac{1}{k}\left(M_{a}\left(q_{a}\right) \ddot{q}_{a}+g\left(q_{a}\right)\right)\right) .
$$

An industrial robot has, in general, six degrees of freedom (6 DOF). However, here we will use only joint 1-3 (not the wrist joints). The following states are used

$$
x_{t}=\left(\begin{array}{lll}
q_{t} & \dot{q}_{t} & \ddot{q}_{t}
\end{array}\right)^{T},
$$

where $q_{t}=\left(\begin{array}{lll}q_{t}^{1} & q_{t}^{2} & q_{t}^{3}\end{array}\right)^{T}$ is the arm angle information from the first 3 axes in Fig 1 and $\dot{q}_{t}$ is the angular velocity and $\ddot{q}_{t}$ is the angular acceleration at time $t$. We use the following state space model in discrete time

$$
\begin{aligned}
x_{t+1} & =A x_{t}+B_{w} w_{t}, \\
y_{t} & =h\left(x_{t}\right)+e_{t},
\end{aligned}
$$

where

$$
A=\left(\begin{array}{ccc}
I_{3 \times 3} & T I_{3 \times 3} & T^{2} / 2 I_{3 \times 3} \\
\mathcal{O}_{3 \times 3} & I_{3 \times 3} & T I_{3 \times 3} \\
\mathcal{O}_{3 \times 3} & \mathcal{O}_{3 \times 3} & I_{3 \times 3}
\end{array}\right), B_{w}=T I_{9 \times 9},
$$

and $T$ is the sampling time.

We assume knowledge about the probability densities for the process noise, $w_{t}$, and measurement noise, $e_{t}$. The observation relation is given by

$$
h\left(x_{t}\right)=\left(\begin{array}{c}
q_{m, t} \\
\ddot{\rho}_{t}
\end{array}\right),
$$

where $q_{m, t}$ is the measured motor angle and where $\ddot{\rho}$ is the Cartesian acceleration vector in the accelerometer frame, Fig 1. The motor angle $q_{m, t}$ is computed from the arm angle using (12). The kinematics [19] of the robot is described by a non-linear mapping

$$
\rho_{t}=\mathcal{T}\left(q_{t}\right),
$$

and the Jacobian is defined as

$$
J(q)=\frac{\partial \mathcal{T}(q)}{\partial q}
$$

The following relation relates the Cartesian acceleration with the state variables

$$
\ddot{\rho}_{t}=J\left(q_{t}\right) \ddot{q}_{t}+\left(\sum_{i=1}^{3} \frac{\partial J\left(q_{t}\right)}{\partial q_{t, i}} \dot{q}_{t, i}\right) \dot{q}_{t},
$$


where $q_{t, i}$ is the $i$ th element of $q_{t}$.

For the PF the nonlinear measurement relation can be applied directly. However, for the EKF it has to be linearized. In the model we do this symbolically, using a symbolic language and then auto-generate a linearized measurement function. Hence, the code can easily be modified. The arm inertia matrix, $M_{a}\left(q_{a}\right)$, and the gravity computation are not implemented symbolically and have therefore been assumed to be constant in the linearization.

\section{EXPERIMENTS}

The experimental platform is the commercial $\mathrm{ABB}$ IRB1400 industrial robot shown in Fig 1 with the inertial and accelerometer coordinate systems accordingly. Since the purpose of the tracking algorithm is to improve the tool position estimate for ILC, there are no real-time or on-line requirements. So both the EKF and particle filter may be applied on data without any constraint on the calculations. In this paper a preliminary experiment is made to test the two algorithms. In the simulation section some robustness and performance aspects are further developed.

\section{A. Measurements}

Measurements are taken from two different systems. The accelerometer data is collected in a Windows NT computer using a National Instrument data acquisition unit and the torque and motor angles are collected by the robot control system itself. Synchronization is performed using a common signal and the sampling frequency is $2 \mathrm{kHz}$ in both systems. Data is decimated 16 times using a standard anti-alias filter which gives the sample time $T=8 \mathrm{~ms}$.

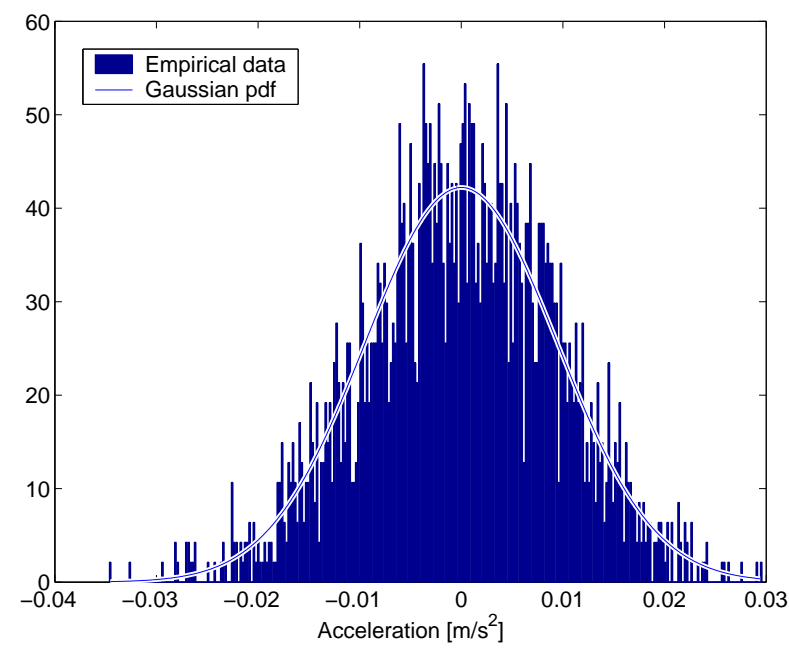

Fig. 3. Empirical acceleration noise pdf, x-channel and an estimated Gaussian pdf.

The measurement noise in the three channels of the accelerometer has been analyzed after decimation and the resulting empirical pdf for the x-channel is shown in Fig 3. The other two channels show a similar behavior. Fig 3 shows the empirical pdf and a Gaussian pdf fitted to data (mean 0 and variance $10^{-4}$ ). The empirical pdf shows a slightly non-Gaussian behavior since more density is located close to the origin. In the EKF the Gaussian pdf must be used while in the particle filter the empirical pdf can be used instead. Preliminary studies of the measurement noise in the angle measurements indicate a non-Gaussian behavior. In this paper it is assumed a Gaussian distribution of the noise because the model of the robot is not included and a more detailed model will be used in future studies.

\section{B. Estimation}

Two different estimators were tested on experimental data. First an EKF and then a particle filter. In the estimation we use the same filter parameters for both methods. The variance for angle measurements is $10^{-9}$ and the variance for the acceleration is 0.1 for the different components. The process noise is 0.3 . These are preliminary design parameters since they must reflect some of the simplifications in the robot model.

EKF. Since the process model is assumed linear the only linearization is in the measurement relation. The linearization is calculated around the predicted estimate of the states. Since the measurement relation uses (20) for the acceleration component, there are many complicated nonlinear expressions. The linearization is therefore performed symbolically so it is straightforward to implement in the EKF given an estimate of the state variable, $\hat{x}_{t \mid t-1}$. The expression is omitted here due to lack of space.

PF. The particle filter can utilize the non-linear model directly, whereas the EKF must use a linearized version. There is also no need to use a Gaussian approximation of the accelerometer pdf and in the estimation the empirical pdf is used. In the estimation presented below the number of particles is $N=4000$.

\section{Experimental results}

The experiment on the robot was a simple linear motion between the points $\rho_{1}=\left(\begin{array}{lll}0.95 & 0 & 1.23\end{array}\right)^{T}, \rho_{2}=$ $\left(\begin{array}{lll}1.08 & 0 & 1.15\end{array}\right)$, with the programmed speed $0.25 \mathrm{~m} / \mathrm{s}$. In Fig 4 the measured angle information, $q_{t}$, is presented.

In Fig 5 the measured and estimated acceleration are shown with two different choices of $R$ in the EKF. A large value implies that the accelerometer is not used, and we compare with the nominal value using the accelerometer. It is worth noting that if we reduce the measurement accuracy in the angle the accelerometer will due to bias error integrate to values that will cause the EKF to diverge. Hence, the accelerometer must be used together with the normal measurement system.

\section{Simulation RESUlt}

Without a laser positioning system it is hard to evaluate the true performance using the experimental data. Therefore we are going to use a MATLAB Simulink 3 DOF model 


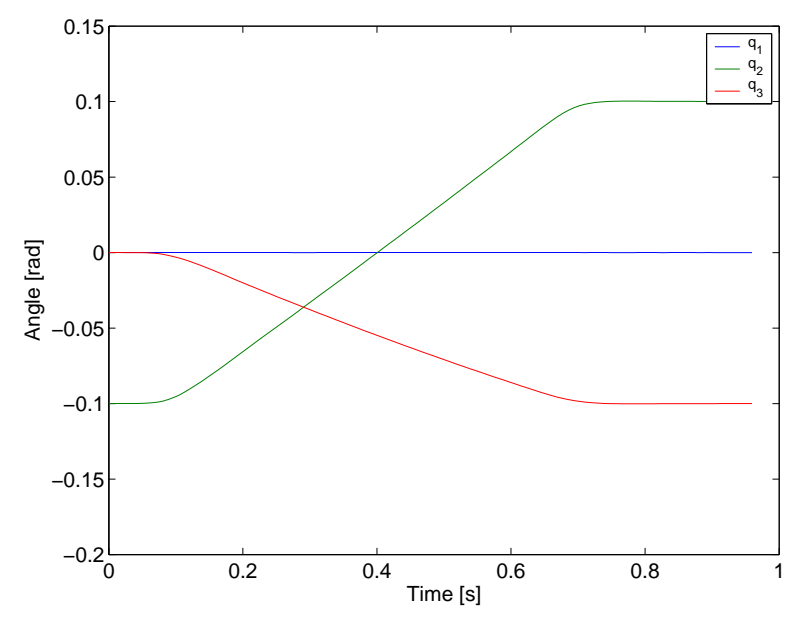

Fig. 4. Measured angles $q_{t}$.
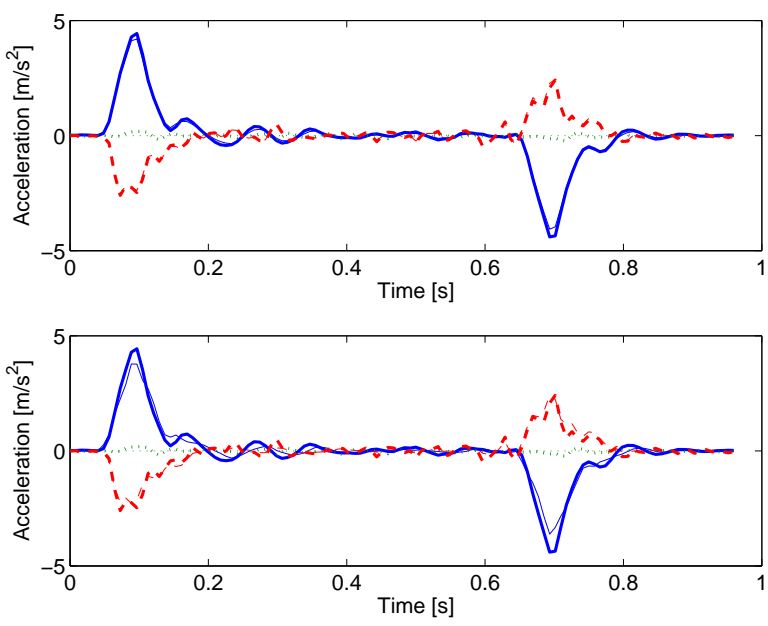

Fig. 5. Measured acceleration (thick line) and estimated acceleration from EKF (thin line), $x$ (solid), $y$ (dotted), and $z$ (dashed). Upper plot using the accelerometer and lower plot without using the accelerometer.

of the robot from [21]. The model is implemented and simulated using the Robotics Toolbox [4].

The simulation model represents a larger physical robot than the ABB IRB1400. The kinematic model is from the ABB IRB7600 and the dynamics is also from a large robot. The robot is stabilized using a PID-controller as shown in Fig 6 and it is assumed that the flexibilities are in the gearboxes (joint flexibilities) as is described in Section IV.

EKF. The EKF is applied to simulation data from the robot model shown in Fig 6 using the reference input shown in Fig 7. The model used in the EKF is the one described in Section IV and compared to the experiment the true arm angles are now available for evaluation of the estimate. The covariance matrices are used as design parameters in the

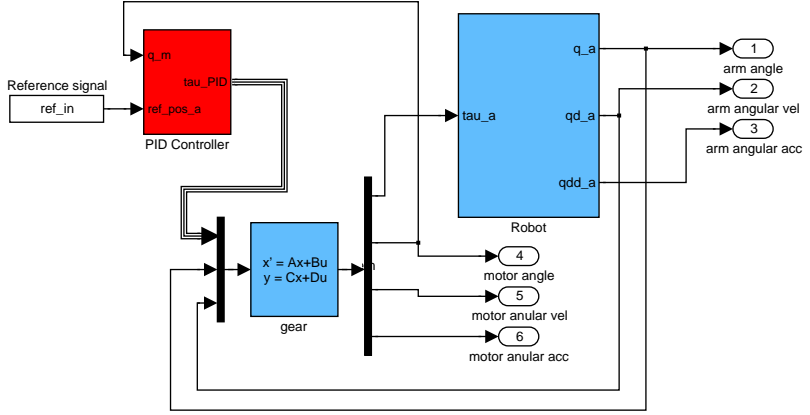

Fig. 6. The Simulink 3 DOF model.

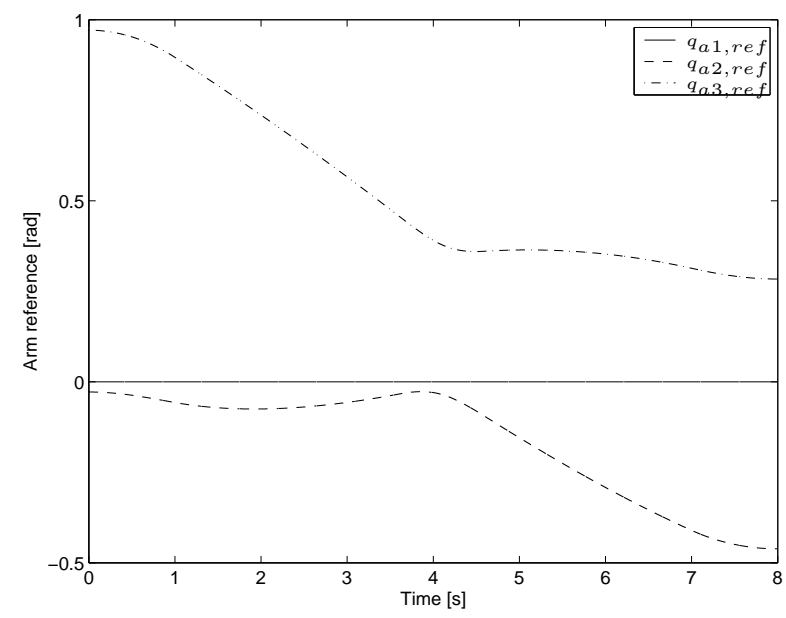

Fig. 7. The arm reference angles in the simulation.

filter and the choice adopted in the simulation is

$$
Q=\left(\begin{array}{ccc}
I_{3 \times 3} & \mathcal{O}_{3 \times 3} & \mathcal{O}_{3 \times 3} \\
\mathcal{O}_{3 \times 3} & I_{3 \times 3} & \mathcal{O}_{3 \times 3} \\
\mathcal{O}_{3 \times 3} & \mathcal{O}_{3 \times 3} & 100 \cdot I_{3 \times 3}
\end{array}\right)
$$

and

$$
R=\left(\begin{array}{cc}
10^{-6} \cdot I_{3 \times 3} & \mathcal{O}_{3 \times 3} \\
\mathcal{O}_{3 \times 3} & 10^{-4} \cdot I_{3 \times 3}
\end{array}\right)
$$

$P_{0}$ is chosen as a diagonal matrix such that it is close to the value achieved at steady state. In Fig 8 the difference between the true arm angle and the measured motor angle is shown for the three joints. A significant part of the low frequency error comes from the gravity acting on the springs in the gear-boxes. This can be compensated for and the remaining error for joint two and three is shown as the thin lines in Fig 9. The error between the true arm angle and the estimated arm angle is also shown in Fig 9. The maximum error compared to using $q_{m}$ is close to 100 times lower and using this estimate for ILC is quite straight-forward but is left for future work.

PF. The particle filter has not yet been tuned for the simulation data but depending on the noise distributions and 




Fig. 8. Difference between true arm angle $\left(q_{a}\right)$ and measured motor angle $\left(q_{m}\right)$ for joints 1 (solid), 2 (dashed), and 3 (dash-dotted).

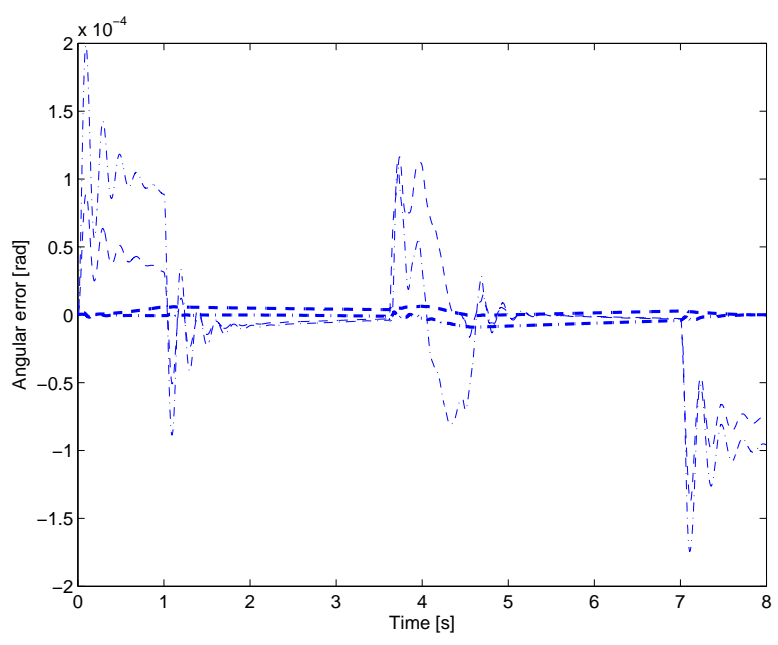

Fig. 9. Difference between true arm angle $\left(q_{a}\right)$ and motor angle $\left(q_{m}\right)$ compensated for gravity (thin lines) for joints 2 (dashed) and joint 3 (dashdotted). Difference between true arm angle and estimated arm angle using EKF (thick lines) for joints 2 (dashed) and 3 (dash-dotted).

also the highly non-linear measurement equation it is likely to improve the result even further compared to the EKF.

\section{CONCLUSIONS}

A multiple sensor fusion approach to find estimates of the tool position by combining a 3-axis accelerometer and the measurements from the motor angles of a commercial industrial robot from $\mathrm{ABB}$ is presented. We formulate the position estimation as a Bayesian problem and propose two solutions. A suboptimal EKF and an approximately optimal estimator using the particle filter method. The algorithms were tested on experimental data from an ABB IRB1400 robot with a three degree of freedom accelerometer attached to the tool and in a simulation of a realistic robot model. The particle filter can handle non-linearities and non-Gaussian noise so the method will handle further improvements using models developed by system identification methods. However, for the simple dynamical model used the EKF perform sufficiently accurate estimates. In the simulation the estimation error decreases to about $1 \%$ of the maximum value achieved when using the motor angle only. Since the intended use of the estimates is to improve on the position using an off-line method, like ILC, there are no real-time issues using the computational demanding particle filter algorithm. It is left for future work to evaluate the robustness of the estimation algorithm and also to do the experiment on the robot using a highly accurate position measurement system for evaluation.

\section{REFERENCES}

[1] B.D.O Anderson and J.B Moore. Optimal Filtering. Prentice Hall, Englewood Cliffs, NJ, 1979.

[2] S. Arimoto, S. Kawamura, and F. Miyazaki. Bettering operation of robots by learning. Journal of Robotic Systems, 1(2):123-140, 1984.

[3] N. Bergman. Recursive Bayesian Estimation: Navigation and Tracking Applications. $\mathrm{PhD}$ thesis, Linköping University, 1999. Dissertations No. 579.

[4] P.I. Corke. A robotics toolbox for MATLAB. IEEE Robotics and Automation Magazine, 3(1):24-32, March 1996.

[5] Inc. Crossbow Technology. Accelerometers, High Sensitivity, LF Series, CXL02LF3. Web. http://www.xbow.com, Jan 2004.

[6] A. Doucet, N. de Freitas, and N. Gordon, editors. Sequential Monte Carlo Methods in Practice. Springer Verlag, 2001.

[7] Leica Geosystems. Laser trackers. Website, http://www.leicageosystems.com/ims/product /ltd.htm, Jan 2004.

[8] N.J. Gordon, D.J. Salmond, and A.F.M. Smith. A novel approach to nonlinear/non-Gaussian Bayesian state estimation. In IEE Proceedings on Radar and Signal Processing, volume 140, pages 107-113, 1993.

[9] S. Gunnarsson and M. Norrlöf. Iterative learning control of a flexible mechanical system using accelerometers. In Preprints of the 6th IFAC symposium on robot control, Vienna, Austria, Sep 2000.

[10] S. Gunnarsson, M. Norrlöf, G. Hovland, U. Carlsson, T. Brogårdh, T. Svensson, and S. Moberg. Pathcorrection for an industrial robot. European Patent Application No. EP1274546, April 2001.

[11] Svante Gunnarsson and Mikael Norrlöf. Iterative learning control of a flexible robot arm using accelerometers. In IEEE Conference on Control Applications, Taipei, Taiwan, Sep 2004.

[12] A.H. Jazwinski. Stochastic processes and filtering theory, volume 64 of Mathematics in Science and Engineering. Academic Press, 1970.

[13] R. E. Kalman. A new approach to linear filtering and prediction problems. Trans. AMSE, J. Basic Engineering, 82:35-45, 1960.

[14] R. Karlsson. Simulation Based Methods for Target Tracking. Licentiate Thesis no. 930, Department of Electrical Engineering, Linköping University, Sweden, Feb 2002.

[15] K. L. Moore. Iterative Learning Control for Deterministic Systems. Advances in Industrial Control. Springer-Verlag, London, 1993.

[16] M. Norrlöf. Iterative Learing Control. Analysis, design and experiments. $\mathrm{PhD}$ thesis, Linköping University, 2000. Dissertations No. 653.

[17] M. Norrlöf. An adaptive iterative learning control algorithm with experiments on an industrial robot. IEEE Transactions on Robotics and Automation, 18(2):245-251, April 2002.

[18] M. Norrlöf and S. Gunnarsson. Experimental comparison of some classical iterative learning control algorithms. IEEE Transactions on Robotics and Automation, 18(4):636-641, August 2002.

[19] L. Sciavicco and B. Siciliano. Modelling and Control of Robot Manipulators. Springer, 2000.

[20] M. Östring, Svante Gunnarsson, and Mikael Norrlöf. Closed loop identification of an industrial robot containing flexibilities. Control Engineering Practice, 11(3):291-300, Mar 2003.

[21] C. Svensson. Multivariable control of industrial robot. Masters Thesis LiTH-ISY-EX-3506-2004, Department of Electrical Engineering, Linköping University, 2004. In swedish. 


Avdelning, Institution
Division, Department
Control \& Communication
Department of Electrical Engineering



URL för elektronisk version

http://www.control.isy.liu.se

\begin{tabular}{|c|c|}
\hline $\begin{array}{l}\text { ISBN } \\
\ldots \ldots \ldots \ldots \\
\text { ISRN }\end{array}$ & .... \\
\hline $\begin{array}{l}\text { Serietitel och serienummer } \\
\text { Title of series, numbering }\end{array}$ & ISSN \\
\hline LiTH-ISY-R-2613 & \\
\hline
\end{tabular}

Titel

Title Bayesian Position Estimation of an Industrial Robot using Multiple Sensors

Författare

Author

Rickard Karlsson, Mikael Norrlöf,

\begin{abstract}
Sammanfattning
Abstract

A modern industrial robot control system is often only based upon measurements from the motors of the manipulator. To perform good trajectory tracking on the arm side of the robot a very accurate description of the system must therefore be used. In the paper a sensor fusion technique is presented to achieve good estimates of the position of the robot using a very simple model. By using information from an accelerometer at the tool of the robot the effect of unmodelled dynamics can be measured. The estimate of the tool position can be improved to enhance accuracy. We formulate the computation of the position as a Bayesian estimation problem and propose two solutions. The first solution uses the extended Kalman filter (EKF) as a fast but linearized estimator. The second uses the particle filter which can solve the Bayesian estimation problem without linearizations or any Gaussian noise assumptions. Since the aim is to use the positions estimates to improve position with an iterative learning control method, no computational constraints arise. The methods are applied to experimental data from an ABB IRB1400 commercial industrial robot and to data from a simulation of a realistic flexible robot model, showing a significant improvement in position accuracy. .
\end{abstract}

Nyckelord

Keywords

extended kalman filter, particle filter, robotics, accelerometer 